\title{
Correcting Some Misinterpreted Concepts and Phenomena in Classical and Relativistic Mechanics (Foundational Concepts and Related Phenomena, Generally)
}

\author{
Sheima'a Thiyab Attiyah Al-Uboodi \\ Dentistry Faculty, Dijlah University College, Baghdad, Iraq \\ Email: sheimaathiab@yahoo.com
}

How to cite this paper: Al-Uboodi, S.T.A. (2017) Correcting Some Misinterpreted Concepts and Phenomena in Classical and Relativistic Mechanics (Foundational Concepts and Related Phenomena, Generally). Open Access Library Journal, 4: e3234. https://doi.org/10.4236/oalib.1103234

Received: November 16, 2016

Accepted: November 11, 2017

Published: November 14, 2017

Copyright (๑) 2017 by author and Open Access Library Inc.

This work is licensed under the Creative Commons Attribution International License (CC BY 4.0).

http://creativecommons.org/licenses/by/4.0/

\begin{abstract}
The topic undertaken in this paper (and probably in some other papers if required for possible future debates) is multi-branched and with various interrelations. It is concerned with the identification and correction of miscellaneous misinterpreted concepts, and with what may appear as some embezzling phenomena, in classical and relativistic mechanics. These include revising the concepts of the independent or dependent existence of matter and its properties, causality and the caused and uncaused states and the fallacy of demanding a cause for the uncaused, relativity of "distance, motion, and interaction" when erroneously absolutised, inertia as an alleged cause of continuation of motion, the imagined fictitious force replacing an overlooked real force, weight under a contact force and unrelated to gravity, the process of weighing based on one of the three effects of a contact force and the catalytic role of gravity in weighing, imparting both rotation and bulge to bodies by inner real centripetal contact forces and not by outer fictitious Newton's absolute space or Mach's cosmic masses, the pendulum oscillation of a body as alternating angular displacement imparted by a contact force irrespective of gravity, balance of the rotating top by dissipating the angular displacement effect by rotation, the relativistic principle of equivalence as absolutised relativities of motion and interaction, ascribing two equal gravitational and inertial masses to a single body,... etc. So far these concepts and phenomena do not seem to have been interpreted correctly in the light of the existing well-established mechanical principles. The identification and correction of the contradictions of these concepts and phenomena with the said principles constitute the subject-matter of this work as will be explained in sections [1] [2]. The "fallacies" or "misinterpretations" of the concepts and phenomena mentioned abovefall
\end{abstract}


under the three categories of the general classification below: 1) Relativity ot distance, motion, and interaction erroneously replaced with absoluteness in some mechanical concepts and phenomena (absolutised relativities). 2) The two forces and three effects of mechanics causally misrelated in some concepts and phenomena (misrelated causalities). 3) The basic mechanical properties ot matter (separate existence, state of motion, and interaction) erroneously ascribed to non-material space, and time, and the related principles of existence, (misplaced properties of space and time). The misinterpretations (fallacies) classified above are contained in, or based on the works of Newton and Einstein. These two scientists enjoy almost untouchable high scientific prestige, but this work will not recognize such a thing as a scientist who is absolutely immune against committing any errors.

\section{Subject Areas}

Classical Mechanics

\section{Keywords}

Revision of Some Mechanical Concepts

\section{Introduction: On the Damaging Role of False Prestige}

For identifying and correcting the miscellaneous misinterpreted mechanical concepts and phenomena under the three categories above, an abbreviated introductory coverage of the relevant theory will be first dealt with in "Section 1: Essentials..." where emphasis is laid on the underlying principles. Some concepts will have to be subjected to revision and some of the fallacies will be handled within the said revision in this section for convenience and brevity. The detailed coverage of the other fallacies will then follow in "Section 1: Examples...".

One point about this work to be emphasized right from the outset is that, generally speaking, this paper introduces no new alternative principles to replace the existing ones, and only concerns itself with the said faulty concepts, i.e., with the contradictions of the topics (enumerated in the abstract) with the existing principles themselves. No computations, drawings, or tables need be quoted for this work since the said principles remain basically unchanged and are assumed to be well-known to the reader. The principles will be referred to by their names, and if necessary, by a few abbreviated identifying descriptions so as to keep the paper within a reasonable number of pages. The fallacies or misinterpretations of the afore-mentioned topics are to be handled, not separately, but within the above combinations or categories according to their various interrelations. This means that some fallacies will be encountered more than once under different names, and their repetition is necessary since the interrelations they exchange in such combinations are considered every time from a different angle or in a dif- 
ferent direction.

Another point also worthy of emphasis in advance is that the scientifically-minded reader cannot accept viewpoints dictated merely by scientists' prestige as undisputable facts, no matter how great the scientists and their achievements might be, but demands a thorough analysis and investigations that prove or refute these viewpoints and reach their underlying truths. As Mach once put it: "to reach the truth it is necessary to put, from time to time, everything in doubt" [1]. The alerted reader has it always stressed in mind that "even the greatest minds are likely to overlook or misunderstand the simplest truths and make untrue interpretations". A scientist well informed and very competent in a certain field can be very ignorant and completely incompetent in other fields. Without this belief firmly held in mind the reader need not take the trouble of reading this paper and of investigating the identified fallacies and their proposed corrections. We should be in the lookout for these fallacies which originate from classical mechanics where they are deeply lodged and prevent them from dominating any "modern" theories. We should guard against physics getting misled unknowingly by these fallacies into gradual metaphysicization in no little part of it.

When disputing with ideas of high prestige a paper submitted to a journal for publication will probably be encountered with attempted omission by the journal. This is especially the case if the journal is not fully aware of the possible trueness or untrueness of the contents of the paper. In this case the journal may tend to defend, so it believes, its scientific reputation by ignoring the paper altogether, thereby confiscating the possible trueness of the paper and eradicating the opportunity of its examination and confirmation by other thinkers. However, such an attitude by the journal would hardly be justifiable since a published paper contains no true or untrue ideas introduced by the journal itself but only what is introduced by the author. The undeserved prestige of false ideas can be so destructive as to discard any proposed corrections. True, we have become civilized enough not to sentence to death a Socrates by poisoning, burn a Jordano Bruno with his books, or proscute a Galileo, but we still to date occasionally do the same misdeed not to the lives of thinkers but to their thought. It is needless to say that with the said cruelty of the past times it was not so much the lives of some individuals as their thought that was aimed at as the final result. The newly introduced sound ideas and corrections, no matter how long they are ignored because of false prestige, will definitely survive at the end, and the corresponding incorrect ideas will inevitably be discarded from science together with their undeserved prestige.

Finally, this paper is not presented as an accomplished work so much as an invitation extended to the interested readers to meet on the pages of this journal and exchange their views on what will now be some debated scientific matters. Any corrections or additions resulting from this paper or from the anticipated debates should by no means be understood to undermine the scientific prestige 
of the relevant famous figures but rather to constitute an integration with, and a continuation of, their efforts, and as an added contribution, no matter how small it might be, to the huge scientific heritage of mankind.

\section{Essentials: The Basic Mechanical Principles Underlying the Correction of the Misinterpreted Concepts and Phenomena Identified in This Work}

In the relevant text-books the principles underlying the concepts and phenomena we are concerned with in this work, are dispersed in numerous sections allocated to them. They are so widely separated that they cannot be readily contrasted with each other. Collecting these principles together in one section (as has been done in this one) will ensure clearer visibility in guiding the researcher through such a diverse mixture of closely related ideas.

\subsection{Relativity of Distance, Motion, and Interaction, Erroneously Replaced with Absoluteness in Some Mechanical Concepts and Phenomena (Absolutised Relativities)}

This paper asserts, among other things, the "relativity" of distance, motion, and interaction, in contrast with the "absoluteness" that is erroneously attached to these three properties in certain cases. In this work "absoluteness" and "relativity" are used in the following meanings:

1) A body does not possess a distance as one-sided (monistic) property characterizing it independently of the other bodies, and is not destined to possess a unique (single) distance; a body necessarily reciprocates a distance with a second body as two-sided (dualistic) relation and contributes to a multiplicity of distances(as many as the other bodies). Furthermore, a distance never undergoes separation or decomposition of its two-sidedness into two one-sided components (like action and reaction), but always retains the oneness (inseparability) of its two-sidedness. Distance can, of course, be viewed in two positive and negative directions but not separately from one another (neither one exists without the other). The above stated absoluteness and relativity, each with its three distinguishing elements, will become much clearer when Examples of the corresponding fallacies are considered in this and the next sections. In contrast with the untrue concept of absolute distance [distinguished by at least one of the three fallacies: one-sidedness (monisticity), singleness (uniqueness), and separated two-sidedness] there exists the true concept of relative distance (characterized by two-sidedness, multiplicity, and inseparability of the two-sidedness). The two terms coined above of absoluteness and relativity apply not only to distance but also to motion and interaction as shown below.

2) From distance, relativity as defined above necessarily passes over to motion for the simple reason that motion is a changing distance.

3) In mechanics, force (interaction) is not known by its nature but by the effects it produces [2]. Recall the fundamental law of mechanics $[F=m a]$. From motion, relativity necessarily passes over to acceleration for the simple reason 
that acceleration is a changing motion. Consequently, interaction being the product of mass and acceleration $[\mathrm{m} \boldsymbol{a}]$ acquires its relativity (dualisticity, multiplicity, and inseparable two-sidedness) from acceleration. However it must be stressed here that two gravitating bodies do not reciprocate one two-sided acceleration relative to each other (as are the cases of distance, motion, and interaction), but two two-sided accelerations of the two bodies relative to their "commoncenterofgravity". The two accelerations are distinct from one another, but still two-sided and inseparable (i.e., neither acceleration exists without the other one).

One of the goals of this work is to identify and eradicate the misinterpreted mechanical concepts and phenomena involving distance, motion, and interaction with their relativity erroneously replaced with absoluteness. These include the three properties (distance, motion, and interaction) distorted by any of the three fallacies of (monisticity, uniqueness and separated two-sidedness). These "absolutised relativities" are not so frequently encountered in connection with distance, being often self-evident in this case. However in the case of motion and interaction these same fallacies defy the thinking mind by appearing as if they were questions that make sense (with their erroneousness completely overlooked). However the insignificance of these questions and the ambiguity of the concepts associated with them will be resolved in this paper.

Today's physics, and the relativistic equivalence principle in particular, introduce extremely absolutised relativities of motion and interaction: "gravity exists and at the same time does not exist relative to different inside and outside observers, and the same goes for the acceleration imparted by gravity" [1] [3] [4]. This would mean subjective existence (dependent on observers) and not objective existence (independent of observers) of gravity and freefall acceleration. Actually observers only come to reveal the already existing gravity and acceleration that are independent of the observers' very existence. If gravity and freefall acceleration were to exist and at the same time not to exist relative to different observers, or more generally, if physical phenomena were to assume certain forms here and now but different forms in different places or at different times, nature would not be governed by physics (i.e., by cognizable laws), but by non-cognizable chaos, or "metaphysics".

\subsection{The Two Forces and Three Effects of Mechanics Misrelated Causally in Some Concepts and Phenomena (Misrelated Causalities)}

These are the fallacies of attributing the two mechanical effects of deformation and angular displacement, not to the contact force where they actually belong, but to irrelevant gravity simply because one of the two coexisting forces (gravity) is emphasized while the other (contact force) is overlooked. Ridding mechanics of the misrelated causalities is the second goal of this work which goal will be clear while and after clarifying the following essentials: 
1) Gravity-dependent and gravity-independent contact forces: Mechanical concepts and phenomena involve two distinct forces (gravitational and contact forces) and three distinct effects [rectilinear acceleration, deformation, and (alternating torque, or angular displacement, or angular oscillation)]. Some contact forces are gravity-dependent, i.e., they arise from the action of gravity on an ordinary body in contact with the gravitating body and vanish when contact between the two bodies ceases. The surface constraining force is one such example. Other contact forces are gravity-independent, as in the case of decelerated motion of a body in a resisting medium, or the accelerated and decelerated motions under forward and backward rocket thrust, respectively.

2) The two forces and three effects, what causes what? In the terrestrial environment where the prevailing condition is that the astronomical object (planet earth) acts in its vicinity on non-astronomical objects (ordinary bodies with comparatively negligible masses), gravity imparts only freefall acceleration and no deformation or angular displacement to the body it acts on. On the other hand a contact force (engine thrust, say) imparts all the three effects of acceleration, deformation, and angular displacement, not one at a time but all at the same time. The acceleration is determined by the resultant of the two gravitational and contact forces but the deformation and angular displacement are determined by the contact force only irrespective of gravity. This distinction between the gravitational and contact forces should be born in mind so as to avoid possible confusion and ambiguity and attain proper understanding of the mechanical phenomena.

3) The case of counterbalance of the gravitational and contact forces: A special case of the pervious situation is when gravity is counterbalanced by a gravity-dependent or a gravity-independent contact force. The equal and opposite gravitational and contact forces acting on a body cancel each other only with respect to "acceleration", but the contact force remains active and produces "deformation" and "angular displacement" which do not arise from gravity but from the contact force even when the latter is gravity-dependent(owes its existence to gravity). Deformation and angular displacement are effects produced by contact forces (such as: surface constraints, pressures, tensions, impact forces, friction, fluid buoyantforce,... etc.) irrespective of whether these forces are generated by gravity or by engine thrust. Where the contact force is generated by gravity the two effects owe their existence to the contact force and not to gravity although the former owes its existence to the latter. Stating it differently, the daughters (effects) are delivered by mother (contact force) and not by grandmother (gravity) although the mother is delivered by the grandmother.

One should guard against attributing to gravity what actually belongs to the contact force. This special case of distinction between the two forces should also be born in mind to achieve clear visibility in analyzing mechanical problems.

4) Real and fictitious forces and accelerations: Given two bodies then they can be either interacting with each other through gravitational or contact forces 
or non-interacting. Motion of "twointeractingbodies" undergoes a "realacceleration" under a "realforce" (gravitational or contact forces). On the other hand, motion of "twonon-interactingbodies" undergoes either "non-acceleration" under "noforce" (such as when the two non-interacting bodies are in free space or in free fall under gravity), or a "fictitiousacceleration" under a "fictitiousforce" (as when one of the two non-interacting bodies, an elevator, say, is interacting with a "third body by a contact force" [the third body can be the gases exhausted at a certain rate in the opposite direction from a rocket). The first and third bodies (elevator and exhausted gases) are "interacting by contact" and their motion undergoes a "realacceleration" under a "realcontactforce". On the other hand the first and second bodies (elevator and a dropped body) are "non-interacting" and their motion undergoes a "fictitiousacceleration" under a "fictitiousforce". The fictitious acceleration and force are actually non-existing and are just imagined images of the real ones that are often overlooked].

Consider two sets comprising three bodies in each set: [earth, elevator and body (B) dropped inside the elevator], and [free body in empty space, elevator and body (B)]. Body (B) has two distinct motions in each set: relative to earth and to the elevator in the first set, and relative to the free body and to the elevator in the second set. Not realizing their being different motions, the equivalence principle replaces the first motion with the second in each set, and speaks of "elimination of gravity by freefall (i.e., by acceleration of the elevator under gravity) in the first set", and of "creation of gravity by acceleration (i.e., by acceleration of the elevator under a contact force) in the second set". The two motions in each set exist objectively and separately from one another although they have one body (B) in common. It is an absurd idea to regard the two motions replaceable with one another in a "principle of equivalence", as if they were one monistic motion of body (B) in both sets.

5) The deformation effect under contact forces irrespective of gravity: Consider three identical elastic helical springs suspended from a ceiling by strings fastened to the springs at different points of application (an upper, lower, and middle points). The first spring will be stretched, the second compressed, and the third compressed in its upper half but stretched in the lower one, respectively. The changes in the deformations of the springs must be due to something changing. Gravity is constant and exerts its action-at-a-distance on all the point masses of the springs directly (i.e., not by transmission of internal stresses through contacting layers of the point masses of the body) and cannot be the cause of the changes in the deformations of the springs. These changes are caused by the equal tensions through the strings (contact forces) due to the changes in their points of application associated with the contact forces and not with gravity.

a) More on the imagined fictitious force replacing an overlooked real force: In the example of the three elastic springs discussed above, an observer imagined to be situated at each of the points of application of the contact forces 
will see that the other ends of the springs are apparently "pushed away from him" or "pulled closer to him" by an "assumed fictitious force" equal and opposite to the "overlooked real force". In the same sense the point masses of a rotating elastic body appear to be pushed outwardly by "assumed fictitious centrifugal forces" when they are actually pulled inwardly by the "overlooked real centripetal forces". Being contact forces, the centripetal forces produce both acceleration and deformation (rotation and bulge) with no need to postulate centrifugal forces to account for the bulge.

b) The concept of weight associated with contact forces irrespective of gravity: Does a body at the earth's surface (i.e., in contact with it) owe its weight to gravity or to the surface constraining force? There is no connection whatsoever between gravity and the phenomena associated with deformation and angular displacement for the simple reason that these two effects are not produced by gravity. Contrary to the usual erroneous belief, weight arises not from gravity but from a contact force irrespective of whether the latter be gravity-dependent (as the constraining force which itself arises from gravity) or gravity-independent (as engine thrust). The pilot of a fighter jet plane can make acrobatic movements (sudden climbing, diving, turning, and overturning) and feel his own weight as well as the weights of his control levers heavier or lighter according to the changes in the thrust or inairdrag (a contact force) whose changes are brought about by changing the plane's orientation. Again it is the changing contact force and not constant gravity that causes the changes of weight. Finally, even from a mere linguistic viewpoint the well known expression "weightlessness in free fall" means weightlessness while in acceleration under gravity, or weightlessness while gravity is acting, which means that gravity imparts no weight to the body it acts on. As a sensation weight is but the deformation exerted by the internal stresses of a contact force.

c) The process of weighing based on one of the three effects of a contact force and the catalytic role of gravity in weighing.

Dropped at the same location bodies of different masses undergo the same acceleration under gravity (Galileo's principle). Consequently "measuring a mass based on measuring its acceleration under gravity" is not possible. Since gravity produces no deformation and no torque it follows that gravity is irrelevant to measuring a mass, or (which is the same thing) to weighing a force (the latter being mass times the already known constant acceleration due to gravity). On the other hand, all the three effects under a contact force can serve as a basis for weighing. "Weighing by measuring acceleration under a contact force" (under a rocket thrust, say) is possible in principle, but hardly practical, since this would mean an experimenter carrying a yardstick and a clock and flying to overtake the accelerating mass to be measured. The "spring balance" measures the "deformation" imparted to the elastic springs by the contact force (surface constraining force generated by earth, or by a space ship accelerating under rocket thrust). And the "beam balance" is counterbalanced by the "two equal 
and opposite torques" by the contact forces on the mass to be measured (which is placed in one of the two pans) and on the standards of weight (placed in the other pan). Since the contact force produces all the three effects at the same time the difficulty of weighing arising from acceleration also arises in principle in weighing by measuring the other two effects (deformation and torque). Fortunately gravity comes here to play its important catalytic role of counteracting the contact force with respect to acceleration but not with respect to the two other effects, and the difficulty arising from acceleration is over, leaving the process of weighing based on the other two effects of deformation and torque.

6) The angular displacement effect attributable to contact forces irrespective of gravity: As already mentioned, in addition to the "rectilinear acceleration" and "deformation" a body undergoes a "torque" as the third mechanical effect under a contact force. Associated with this third effect are the concepts of "center of mass" of the body, "point of application, or of action", "line of action", and "plane of action" of the contact force. The plane of action is determined by the center of mass and the line of action. These concepts arise only in connection with contact forces, and are unrelated to gravity. In acting on a body gravity does not act on contacting layers of point masses of the body. Gravity acts directly by exerting its "action-at-a-distance" on all point masses of the body (as points of application) which is why it imparts "no deformation" to the body it acts on.

a) The pendulum oscillation phenomenon as an alternating angular displacement under a contact force irrespective of gravity: The resultant gravitational force (of the individual component forces exerted on the point masses of a body) is assumed to pass through the center of mass which is another way of saying that gravity exerts "no torque" to a body in the terrestrial condition where the dimensions of the body are comparatively negligible. In free fall (i.e., in the presence of gravity and absence of contact forces), a body does not undergo rotation, and its initial rotation, if any, will remain unchanged under gravity. A contact force, on the other hand (unless the line of action of the force passes through the center of mass of the body) has the [tendency to impart an "angular displacement" to the body, in the "plane of action" such that the "centerofmass" of the body is thrown on the "line of action" of the force behind the "point of application"]. This means that a torque is exerted about an axis perpendicular to the plane of action to oscillate the body about the two sides of the line of action with the point of application (suspension) held stationary permanently or momentarily. In resisting media the third effect is damped away such that the final result will be a certain "angular displacement" of the body relative to its initial orientation. In ideal conditions where the resistance to the angular displacement is practically negligible, the effect will result in "angular (pendulum) oscillation". In rotating the body, during its first quarter cycle from the initial orientation to the position of coincidence of the center of mass with the line of action, the torque decreases gradually to zero but rotation con- 
tinues increasing to maximum. During the second quarter the torque increases to maximum in the opposite direction impeding the rotation which decreases to zero on completing the first half cycle. The second half cycle is then effected in the same manner in the reverse direction.

b) Therotating top balance by dissipating the angular displacement effect by rotation:

Whereas the angular displacement effect imparted to a body by a contact force accounts for the pendulum oscillation of the body, the dissipation of this same effect by rotation prevents a rotating top from falling down and keeps it in balance even in a tilted axis of rotation. The dissipation of the angular displacement by rotation also keeps the gyroscope pointing to a fixed direction as a reference for changes in the directions of planes and ships. It also prevents the deviation of projectiles from their specified trajectories under local influences of air.

Consider the angular displacement imparted during an infinitesimal period to a "certain point mass" of the top in the plane containing this point mass and passing through the axis of rotation, which plane is rotating rigidly with the top. If this angular displacement is, initially say, "clockwise", then it becomes "anticlockwise" after half a revolution of the top and counterbalances the clockwise angular displacement imparted to the "opposite point mass" during an infinitesimal period after a half revolution of the top, and this goes for all the point masses of the rotating top. That is how dissipation of the angular displacement effect is performed. Dissipation of the angular displacement by rotation is similar to the case of a mass acted upon by a force that reverses its direction rapidly and repeatedly. The mass will accelerate in neither direction and remain stationary under such a rapidly alternating force.

The second goal of this work is to identify and correct the causalities "misrelated" in some mechanical concepts and phenomena. In addition to the ones already identified and corrected above these fallacies also include the fallacy demanding a "cause for the uncaused, i.e., the principle of inertia", the fallacy of an "effect imparting a cause", and an "effect imparting another effect" as in the bulge of rotating bodies.

\subsection{The Basic Mechanical Properties of Matter (Separate Existence, State of Motion, and Interaction) Erroneously Ascribed to Non-Material Space, and Time, and the Related Principles of Existence, (Misplaced Properties of Space and Time)}

Matter exists in the already known form of nuclei and elementary particles. It may also exist in the still unknown form of fields, gravitational waves, and gravitons. Action-at-a-distance through empty space (without the mediation of fields) was doubted by Newton himself, and Einstein spent his last years attempting to formulate a "unified field theory" but without success. We are not concerned here with topology or with the recent complicated mathematical constructs of space and time, but with the simple intuitive understanding of space 
and time as expressed by Newton. There are three central viewpoints or principles that can be thought of in connection with the existence, motion, and interaction of matter and its basic properties, space and time. The three viewpoints can generally be concluded from the works of Newton, Einstein, and this work: 1) whether the properties of matter can be endowed with separate or inseparable existence from matter', 2) whether the properties possess a state of motion as matter does, and 3) whether the properties interact with matter as matter does with itself.

The following essentials may be recalled:

1) The classical principle of existence: Newton founded the universe on the three pillars of matter, space and time, each endowed with separate existence. According to this "classical principle of existence" if any of the said three pillars were to be eliminated the remaining ones would retain their separate existence unaffected [4]. Newton also ascribed a state of motion to both of space and time. He believed space to be at absolute rest (relative to certain fixed stars in our Milky Way galaxy) and time in motion (relative to what?, at what rate?, and in what direction?). Furthermore, in spite of its being non-material Newton's absolute space became capable of acting on matter (as in the phenomenon of bulge of rotating bodies) contrary to Newton's third law of action and reaction according to which it is matter that acts and matter that is acted upon. Also a Russian astronomer thought of the possibility of generating energy from the passage of time.

2) The relativistic principle of existence: In the special relativity theory where matter and field do not exist, the space-time continuum does exist and is flat (Euclidian) [4] [5]. In the general relativity theory, on the other hand, matter does not attend the scene carrying space-time continuum as a property but comes to act on, and impart curvature (non-Euclidity) to, an already existing space-time. In relativity space and time are inseparable from one another, but they are separate from matter. This "relativistic principle of existence" is in full conformity with the classical principle in ascribing existence to space and time separately from matter. In relativity both space and time are endowed with motion as is the case in Newton's mechanics. Furthermore space contracts with motion and time dilates with motion. Relativity also speaks of an opposite action on matter by curved space-time with the curvature imparting geodesic (inertial) motion under no-force in a new relativistic theory of gravitation (i.e., with curved space time acting as a guiding field itself and no longer as a mere container or scaffolding carrying the field as in classical mechanics. Matter at first "curves" space-time and then it becomes "guided" by the curvature! In effect this would mean a material body accelerating itself by itself.

3) The corrected principle of existence: Because of their being non-material, space and time cannot possess any of the basic properties possessed by matter. Space and time cannot be but themselves properties of matter in a kind of existence that is dependent on, and inseparable from the existence of matter that 
possesses the properties. Also according to this "corrected principle of existence" space and time are not moving, and are not at rest either. Space and time are properties with respect to which the state of motion does not arise in the first place. What actually moves is matter possessing properties, and not properties possessed by matter. Finally space and time being non-material cannot interact with matter which would violate Newton's third law of action and reaction between two material bodies. Space and time are, so to say, pieces of nothingness turned into somethingness by being attached to matter as properties. Every physical entity has to be either matter or a property of matter, with no third possibility.

With the totality of its concepts in view classical mechanics seems to be suffering from a number of self-inconsistencies, namely; space and time existing separately from matter, space and time with a state of motion, and space and time interacting with matter. These self-inconsistencies are no less fallacious than the science fiction allegations of the possibility of travelling in time to the past or to the future, or generating energy from the passage of time!

As a third goal this work attempts to rid classical mechanics of the fallacies of the "misplaced properties" associated with the non-material space and time which fallacies accompanied mechanics since its early beginnings.

\section{Examples of the Fallacies of Absolutised Relativities, Misrelated Causalities, and Misplaced Properties of Space and Time}

\subsection{Examples of the Fallacies of Absolutised Relativities (Relativity of Distance, Motion, and Interaction Erroneously Replaced with Absoluteness)}

\subsubsection{The Absolutised Distance Fallacies}

The meaninglessness of these questions is so self-evident that they were not posed previously in connection with distance. As to why such obviously insignificant questions have been posed in the first place in this paper, the answer is as follows: Not all questions make sense. These same insignificant questions appeared as if they were significant to many great minds who posed them in different forms and in different relative relations other than distance, such as (trajectory, motion, and interaction) as will be shown later. Starting with the distance fallacies will simplify the understanding of these fallacies in connection with the other relativities (motion and interaction).

1) The monistic (one-sided) distance fallacy: "How far is Baghdad?" The answer to this incomplete question will also be a question: "Far from what?", unless the second city reciprocating the distance with Baghdad is understood impliedly. Geometrically, distance is the space between "two points" but physically, between "two material bodies", or rather "two point masses". It is these material marks what makes it possible to perform measurement and experimentation with distance (and with the other relativities: motion and interaction). Without specifying both of its sides distance (as well as the other relativities) 
would be immeasurable and turn into "unobservables in principle" i.e., into physically meaningless suppositions. Strictly speaking, the first question speaks erroneously of distance as a monistic (one-sided) property, whereas the answer requires the missing second side to be specified, thereby insisting on the dualisticity (two-sidedness) of distance [which, of necessity, characterizes the changing distance (motion), the changing motion (acceleration), and (interaction), the product of mass and acceleration, as well].

2) The unique (single) distance fallacy. A body $B$ reciprocates the distances $\left(\boldsymbol{d}_{1}, \boldsymbol{d}_{2}, \cdots, \boldsymbol{d}_{n}\right)$ with (n) other bodies $\left(\boldsymbol{B}_{1}, \boldsymbol{B}_{2}, \cdots, \boldsymbol{B}_{n}\right)$. Which one of the $(\boldsymbol{n})$ distances is the distance of body $\boldsymbol{B}$ in reality? Answer: the question is fallacious. A body is not destined to "possess" one distance but "reciprocates" as many distances as the other bodies. All the ( $\boldsymbol{n}$ ) distances exist objectively, independently of one another, and irrespective of body $\boldsymbol{B}$ being a common side in the (n) two-sided distances.

3) The two-sidedness decomposition fallacy of distance (i.e., The fallacy of distance measurement confined to one body under the caged or inside observer condition): "An inside observer cannot determine the distance between a ship and the shore by measurements totally performed inside the ship". This statement was first posed in connection with motion, but we now borrow it in connection with distance for clarification purposes. The statement is very suggestive of fallacious implications. What justifies from the physical point of view the denied access to the second side (shore) of the two-sided distance by caging the observer inside the ship which is only one of the two sides? The "caging condition" can hardly be accepted as a meaningful modern physics as it erroneously changes the "two-sidedness" of a property into "one-sidedness".

\subsubsection{The Absolutised Motion Fallacies}

The meaninglessness of these fallacies is not self-evident and these fallacies were posed by great thinkers as if they were significant questions:

1) The monistic (one-sided) motion fallacy: We are often interested in motions of bodies relative to the earth which serves as the other side common to all the two-sided motions. The linguistic expression of motion will be much lighter if [repeating the word "earth" every time we speak of motion is avoided by dropping the word "earth" only linguistically and not physically].But then the language will be very suggestive of the fallacious monistic and not the true dualistic motion. To alienate this very misleading linguistic fallacy it should be stressed in mind that where earth is not explicitly mentioned, as is often the case, it is always to be understood implicitly, unless another reference body is meant.

2) The unique (single) motion fallacy: Consider three passengers $X, Y$ and $Z$ waiting at a bus stop. The number of $\mathrm{X}$ and $\mathrm{Y}$ arrives and they get on the bus and $Z$ remains waiting for his number. The bus then accelerates from rest to its normal speed. $\mathrm{X}$ is at rest relative to $\mathrm{Y}$ who is sitting beside him in the bus and at the same time in accelerated then inertial motions relative to $Z$ who is still waiting at the bus stop. Is the motion of $\mathrm{X}$ in reality accelerated, inertial, or rest? 
Answer: the question is fallacious. Body $\mathrm{X}$ is not destined to possess a one-sided single motion but reciprocates as many two-sided motions as the other bodies. No motion is a replacement of another, and all the motions are real and independent of one another in spite of the fact that the motions have one common body X. Motion should not be attached monastically to one body X, but dualistically to any two of $\mathrm{X}, \mathrm{Y}, \mathrm{Z}, \ldots$

Consider another example. The trajectory of a ball dropped vertically inside a uniformly and rectilinearly moving train is rectilinear relative to the train but parabolic relative to the station. Is the trajectory of the ball rectilinear or parabolic in reality? The expression preceding the question speaks correctly of dualistic motions and trajectories, but the question changes incorrectly to monistic motions and trajectories. Both trajectories exist objectively, and neither one can be eliminated and replaced with the other, as is erroneously done in the equivalence principle. What exists in reality is not "one motion with two alternative descriptions", but "two motions each with one specified description".

3) The two-sidedness decomposition fallacy of motion (i.e., The fallacy of motion measurement confined to one body under the caged or inside observer condition):

"Motion of a ship cannot be measured by experiments totally performed inside the ship". The statement can be misunderstood to imply that motion is one-sided and unobservable in principle. Which of the many motions the ship reciprocates with the other bodies is to be measured? If it is motion of the ship relative to the shore then what sense does it make to deny access to the shore as the second side of the two-sided motion and permit access only to the first side-the ship? Decomposing a two-sided relation into two one-sided components and discarding one of the components and retaining the other is a fallacy to be found in some concepts as the relativistic equivalence principle which will be considered later.

\subsubsection{The Absolutised Interaction Fallacies}

The fallacies related to interaction are not restricted to the absolutised relativities but extend also to the misrelated causalities and the misplaced properties of space and time.

1) The monistic (one-sided) interaction fallacy. Newton's second law of motion.

This law speaks, not of dualistic (two-sided) interaction reciprocated between two bodies as it should do, but of monistic (one-sided) action on one body. Therefore the form of the law is defective from the descriptive point of view as it reduces the true relative interaction into an untrue absolute one. However it must be stressed that the law is very precise for calculations under the terrestrial condition of negligible masses interacting with massive earth. The acceleration imparted by interaction to massive earth is so trivial that it is practically immeasurable though calculable in principle. This condition makes the defective form of the law (i.e. it's being confined to one body) practically acceptable as a limit- 
ing case. But a reformulation of the law stating the two-sidedness of interaction (gravitational, and a contact force) is possible and necessary.

2) The unique (single) interaction fallacy. The relativistic principle of equivalence as absolutised relativities of motion and interaction.

This principle serves as a foundation for general relativity theory (i.e., for the relativistic theory of gravitation). It involves thought experiments concerned with the motions of a body (B) dropped inside an elevator that is accelerating in two cases: 1) an elevator accelerating under a gravitational force (present gravity and absent contact force) and 2) an elevator accelerating under a contact force in empty space where no matter exists (absent gravity and present contact force). According to the equivalence principle gravity and the freefall acceleration it imparts may and may not be assumed to exist or can be wiped out by freefall (i.e., by acceleration of the elevator under gravity), and created by acceleration (i.e., by acceleration of the elevator under a contact force), relative to observers outside and inside the elevator respectively. These deductions, believed to be correct, are essential for constructing a new theory of gravitation. It is important to note that interaction should not be looked upon as something existing in some place, but as a dualistic property characterizing two material bodies as is the case of distance and motion. In case (1) body (B) is "interacting" with earth (one of the two interacting bodies being astronomical), but "non-interacting" with the elevator (neither of the two bodies is astronomical). The "accelerated motion" of (B) relative to earth under "existing gravity" and the "non-accelerated motion" of (B) relative to the elevator under "non-existing gravity" are two objectively and separately existing motions although they have one body (B) in common. It cannot be deduced that the "existing gravity and accelerated motion between (B) and Earth" can be "eliminated by freefall" of the elevator and replaced with "non-existing gravity and non-accelerated motion between (B) and the Elevator". Neither motion can be discarded and replaced with the other. Being separate from one another, both motions exist together at the same time independently of the observers. The "caging condition" is quite superficial and plays no role in this connection. Likewise it cannot be deduced in case (2) that the "non-existing gravity and non-accelerated motion" of (B) relative to a Free Body in empty space can be "converted by acceleration" of the elevator (under a contact force) into "existing gravity and accelerated motion of (B) relative to the Elevator" for the same reasons already mentioned.

In the equivalence principle distinction should be made between the gravity-dependent and gravity-independent contact forces acting with gravity in two cases that are actually "distinguishable from one another" (contrary to the assertion of the principle). Consider a collection of bodies of different masses placed on a shelf near the ceiling of an elevator. Let the different masses be dropped to the floor one by one. If in a first case the elevator is accelerating in empty space under a gravity-independent contact force then during its fall from 
the ceiling to the floor the dropped body will not be in contact with the force and the latter being "non-changing" will act during the fall on a lesser total mass and impart a greater acceleration. The greater the dropped mass the greater the acceleration. This is obviously distinguishable from the second case of different masses dropped one by one and undergoing the same acceleration in an elevator at rest on the earth's surface. This is due to the "decrease" of both gravity and the constraining force acting on the resting elevator whosetotalmass "decreases" during the fall of the dropped body. If the elevator hovers above the earth's surface under a gravity-independent contact force then the "two cases" are actually "one repeated case" of an elevator whose internal occurrences are determined by the presence of the gravity-independent contact force "irrespective of the absence or presence of gravity". In such a situation we have, not equivalence of two things that have to be dissimilar in some respect, but equivalence of two things that are similar in every respect, or rather equivalence of the same thing with itself which would be an insignificant principle.

Undoubtedly, Einstein was the greatest relativist who asserted the transition from the one-sided (absolute) motion to the two-sided (relative) motion. But he returned back in his equivalence principle from the dualisticity to the monisticity of motion, and thereby violated what he himself asserted. "Einstein's story of motion in the equivalence principle" repeats "Newton's story of inertia" (to be told later). In both stories there is a fallacy refuted in some concepts but unknowingly resumed afterwards in other concepts.

Finally, one may wonder what magic is invested in the "caged or inside observer condition" such that the condition turns the "existing into non-existing" i.e., (gravity and freefall acceleration outside and inside an elevator accelerating under gravity), and turns the "non-existing into existing", i.e., (gravity and acceleration outside and inside an elevator accelerating under a contact force in empty space). From the above arguments it follows that the equivalence principle and its associated caging condition have been refuted.

3) The two-sidedness decomposition fallacy of interaction.

a) Newton's third law of matter-matter interaction (action and reaction): "to every action there is a reaction equal in magnitude and opposite in direction". In spite of its defective original formulation, Newton's third law serves as a principle of matter-matter interaction and handles correctly the concept of interaction as relative property of matter as follows:

i) The two-sidedness (dualisticity) of interaction. There have to be two bodies in order for interaction to be reciprocated, as is the case with distance and motion.

ii) Matterness of the interacting bodies. It is matter that acts, and matter that is acted upon. Action by, or on, nonmatter is not possible according to this law of machanics.

iii) Non-matterness of interaction (interaction being a property of matter, is non-material). 
iv) The two-sidedness of interaction, like that of distance and motion, cannot be decomposed into two one-sided components, but can be viewed in two opposite directions positively and negatively, inseparably from one another. Action and reaction are not two separately existing things, but one two-sided interaction with inseparable two-sidedness. Action and reaction cannot exist one without the other.

b) Comparison of the "two-sidedness of interaction" in Newton's law of universal gravitation, the third law of action and reaction, and the second law of motion.

As an example of the "two-sideness decomposition fallacy of interaction" one can cite Newton's third law of interaction which is usually misexpressed linguistically as law of "action and reaction". Instead of expressing the oneness of the two-sided interaction (whether this be gravity or a contact force), the usual formulation of the third law erroneously decomposes the two-sidedness of interaction into two one-sided action and reaction as if they were separate components of interaction. Also Newton's second law [ $f=m a]$ (i.e., reducing interaction between two bodies to a force on one body) is another example of the "two-sidedness decomposition fallacy of interaction" since it concerns itself only with the action on one of the two interacting bodies. The formulation of the third law should be the same as that of the law of universal gravitation with respect to the two-sidedness of interaction. Also the formulation of the second law should likewise be amended. Reformulation of the three mechanical laws that makes them consistent with respect to the inseparability of interaction will make them properly expressed and more clarified.

c) The "solar system" as a special limiting case of the "binary star system" [6]. Strictly speaking splitting the two-sidedness of interaction is untrue in the general case where the two interacting masses are comparable to each other as in the binary star systems. However such splitting of Newton's second law (concerned with one-sided action) is definitely true as a special limiting case of the third law and of the law of universal gravitation (both of which are concerned with two-sided interaction), and constitutes the foundational law of mechanics for the simplistic reasons it brings about in mathematical calculations. By way of illustration one can mention Kepler's first law of planetary motion which is mathematically derivable from Newton's laws, and according to which "a planet moves around the sun in an elliptical trajectory with the sun at one of the two foci". Going from the "solar system" to the "binary star system" one finds that both of the companions of the latter system have their own "distinctbutinseparable" elliptical trajectories. Only when the mass of one of the companions is negligibly small does the trajectory of the other massive companion degenerate from an ellipse to a focal point, which is the prevailing condition in the solar system as a limiting case (negligible masses of the planets compared to the mass of the sun). It may also be interesting to note that if the size of earth were to contract to that of a small ball, then a book dropped to the ground would move 
around earth in anelliptical trajectory (orbit) as a satellite. It is the collision of the book with the earth's surface what prevents the book from becoming a satellite.

d) Other related fallacies: "escape from gravity" and "disappearance of gravity": Also note in connection with interaction that the term "escape veloci$t y$ " is a somewhat misleading linguistic fallacy. There is no escape from gravity which ceases only at infinity. In circling the earth a satellite moves with the tangential (escape) velocity and is at the same time in a continuous free fall towards the center of the earth without colliding with the earth's surface. This is because the free fall under gravity takes place at a rate equal to the rate at which the surface of the earth curves away due to its spherical form from the tangential line through every point along the trajectory. Consequently the satellite keeps moving at constant altitude at the (escape) velocity although it is continually falling [6]. Another point to be noted is that phenomena such as pendulum oscillation, deformation of elastic bodies, overturning of a body when the vertical projection of its center of mass is moved outside its supporting base, ... are commonly observed at the earth's surface where the constraining force is acting, but they disappear in space flight when the space ship's rockets are turned offand appear when the rocket thrust is applied. From this it is erroneously concluded that "gravity disappears" in the first case (and is created in the second) which is another version of the former fallacy of "escape from gravity". The two fallacies are even contradictory to each other (one retains and the other discards the existence of gravity). The appearance and disappearance of these phenomena is associated with the presence and absence of contact forces independently of the presence or absence of gravity.

\subsection{Examples of the Fallacies of Misrelated Causalities (the Two Forces and Three Effects of Mechanics Misrelated Causally)}

1) Miscellaneous fallacies of the misrelated causalities: Some of the fallacies of attributing to gravity what actually belongs to the contact forces have already been identified and corrected in [Section 1 - 2], such as weight, weighing, pendulum oscillation,... etc. Contact forces whose effects are usually but erroneously attributed to the dominant gravity include the constraining force, engine thrust, pressure, tension, impact force, friction, buoyant force,... etc. As a result numerous fallacies of misrelated casualties are formed. There are also two important fallacies to be detailed below.

2) Causality and the caused and uncaused states. Inertia as an alleged cause of continuation of motion (the fallacy of demanding a cause for the uncaused)

Causality is a basic principle in our scientific thinking. The belief in a physical world that is cognizable and knowable in principle is a prerequisite without which science would evidently be pointless. Cognizability and knowability of the physical world presuppose its causality, for without causality the only alternative 
would be arbitrariness that would turn science into metaphysics that is uncognizable and unknowable. Generally speaking, causality seems to mean that things should remain in steadiness (in an inertial state, in the Newtonian sense) and, no change should occur except as an effect arising from a definite cause. There has to be a cause for every change, but no cause for non-change (steadiness). We shall make two applications of the principle by reviewing the concept of inertia and that of centrifugal force.

Of the two aspects of motion (causation and relativity), only the first aspect attracted the ancients' attention, whereas the second aspect is of a quite recent origin. The ancients believed in rest as an "uncausedstate" and in motion as the corresponding "caused state". In this erroneous intuitive belief, motion including uniform rectilinear motion demanded the continued application of a cause (force) and bodies came down to rest when the cause of motion ceased. It was Galileo and Newton's achievement to realize that uniform rectilinear motion at whatever magnitude including (rest as a uniform rectilinear motion of zero magnitude) is the uncaused state and that acceleration of motion is the caused state. In this new belief force remains uncalled for in connection with uniform rectilinear motion and the role force played in the old belief as the cause of motion is cancelled in the new belief. Force no longer generates motion but only changes an already existing motion that is uncaused (requires no cause for its a priori existence). When force ceases only acceleration ceases and motion continues at its final magnitude. Bodies have no tendency to come down to rest as was erroneously assumed, and whereas they came down to rest by removal of force in the old belief, they now require the timed exertion of force in order to come down to rest in the new belief. But the old intuitive belief was deep-rooted even in a great mind as Newton's. Newton was not completely free from the erroneous belief which he himself refuted! After abolishing force as the cause of uniform rectilinear motion Newton felt a gap left behind and thought that something has to be responsible for the continuation of motion. He ascribed a negative property to material bodies which he termed "inertia" to fill the gap and assume the role of continuation of motion. Force went out and inertia came in. Bodies now move by their inertia which is equivalent to the statement that were it not for inertia bodies would come down to rest, as was the case in the old belief. Newton turned uniform rectilinear motion from a caused state in the old belief into an uncaused state by abolishing force, but he turned it again into a caused state by introducing inertia, and returned to his initial point after looping a vicious circle. The essence of Newton's first law is the motion "being" uniform rectilinear, and not being "kept" as such in the absence or counterbalance of forces. And whereas inertia is a negative property (inertness, inability, powerlessness) that signified absence of forces it has now become an active property and almost a force in itself. The term "inertial force" in which inertia no longer signifies absence of force is now common in the literature. However, we now know that the question why bodies move in uniform rec- 
tilinear motion is a self-inconsistent question demanding a cause for the uncaused, and may only be accepted to mean why bodies do not accelerate. If there has to be a reason why bodies move uniformly and rectilinearly then it is certainly the absence of the external force and not the presence of an internal inertia. This discussion has been on the concept of inertia, one of the early misunderstandings with which Newton's mechanics started, and on which many thinkers worked hard but in vain.

3) Imparting both rotation and bulge to bodies by inner centripetal contact forces, and not by outer Newton's absolute space or Mach's cosmic masses.(The fallacy of an "effect generating a cause", or the "two-effect fallacy").

Consider a "three-element causality" consisting of a single cause $\mathrm{C}$ producing two effects $E_{1}$ and $E_{2}$. A typical example of such acausality is the contact force and the two effects it produces at the same time, acceleration and deformation. In this triple causality both effects $E_{1}$ and $E_{2}$ occur "when and because" $\mathrm{C}$ does. On the other hand each of the two effects occurs "when, but not because" the other effect does. Where cause $\mathrm{C}$ is overlooked and only the effects $E_{1}$ and $E_{2}$ are recalled, and observing that the two effects appear and disappear together then we are driven by our general trends of thought to erroneously postulate a causal connection between them. The three-element causality will then appear as if it were a two-element causality, and the observer will be trapped by what we have called the "two-effect fallacy". By attributing bulge (deformation) to rotation (acceleration) in their interpretations Newton and Mach seem to have been trapped by the said fallacy. They seem to have viewed the problem as follows: When a body rotates it bulges and when the rotation disappears, the bulged is appears too. Posing the problem in this way will strongly but erroneously suggest that rotation causes bulge. The problem should, however, be viewed as follows: "When centripetal forces are acting the body both rotates and bulges and when these forces disappear so do both effects". Only in this way can Newton's Mechanics avoid being self-inconsistent with respect to the phenomenon in question. "Centrifugal forces" should therefore be discarded from mechanics, and with them the ideas of "a space acting on matter", "a forcegenerated by acceleration" [7] and Mach's principle. This will naturally cast no little doubt on the validity of all succeeding ideas founded on, or related to, these fallacies.

Let us note again that the point masses of a rotating elastic body appear as if they were pushed outwardly by centrifugal forces (thought to be exerted by Newton's absolute space, or by Mach's cosmic masses). But then the equal and opposite centripetal and centrifugal forces acting on the same point masses would cancel out and there would be neither rotation nor bulge, which is not the case. The point masses are actually pulled inwardly as "Newtonian couples set in rotation by the tensions of the strings connecting them" (i.e., by centripetal forces of internal origin). The "fictitious centrifugal forces" are the mirror 
image of the "overlooked centripetal forces" (recall the example of the springs compressed and stretched according to the points of application of the applied contact forces). There is no such a thing as a contact force producing only one of its three effects without the others. Being contact forces the centripetal forces produce both acceleration and deformation (rotation and bulge). Newton's absolute space is non-material and cannot interact with material bodies (i.e., cannot exert deformation on them). Also the dispersed cosmic masses would have to propagate their actions to the rotating body at an infinite velocity so as to traverse the different distances and reach the body at the same time, to exert the bulge.

Another version of this same problem that has remained unsolved so far is: $\boldsymbol{O}$ two spheres one rotates and bulges and the other is stationary and undeformed, it is hard to understand how the balls know which of them rotates and hence must bulge. The words are Einstein's. First of all the question of rotation and non-rotation of the spheres can be easily established experimentally by observing the distinguishable motions of a free body. The motion will be "uniform-rectilinear" relative to the non-rotating sphere, but "helical" relative to the rotating one. Secondly, "what the spheres really need to know" is which of them is acted upon by centripetal forces and hence must both rotate and bulge. That is the real solution of the stated problem.

\subsection{Examples of the Fallacies of Misplaced Properties [the \\ Basic Mechanical Properties of Matter (Separate Existence, State of Motion, and Interaction) Erroneously \\ Ascribed to Non-Material Space, and Time]}

1) The fallacies of "existence of the non-existing", and "non-existence of the existing". Motion of bodies dropped inside an elevator is "accelerated and at the same time unaccelerated (inertial)" under a "gravitational field that may and may not be assumed to exist" relative to different inside and outside observers (already reviewed within the equivalence principle).

2) The fallacies of "space without matter" and of "matter without space". When distances are compared space is created, and when motions are compared time is generated. In contradiction to this statement it is commonly believed that space and time contribute somehow to the generation of motion. Actually time does not contribute as input to the generation of motion, but is itself generated by motion as output.

Space is the generalization into three dimensions of the one dimensional distance. Space without matter is equally meaningless as distance between no two bodies. Likewise, matter without space is equally meaningless as two bodies reciprocating no distance. Space (and time) do not possess separate existence but are in a sort of existence that is dependent on, and inseparable from the existence of matter itself, as previously mentioned.

3) The fallacy of ascribing a state of motion to space and time (the space traversing space, and time elapsing time), seem to be absurd ideas. 
4) "The fallacy of interaction between matter and non-matter". The idea of matter-non-matter interaction violates the classical principle of matter-matter interaction (Newton's law of action and reaction). It also violates quantum physics according to which certain interactions take place by exchanging certain elementary particles between the interacting bodies. Space and time being non-material cannot create and transmit, or receive and absorb elementary particles, only matter can. Action by or on non-matter (space or time) seems to be inexplicable in principle and is consequently absurd.

\section{Conclusions}

1) The three mechanical properties (distance, motion, and interaction) possess three relativising factors: (dualisticity, multiplicity, and inseparability of the two-sidedness). The concepts of these properties will be turned into fallacies (misinterpreted concepts and phenomena) by having any of the three absolutising factors attached to them: (monisticity, singleness, and decomposed twosidedness). An outstanding example of these [ $3 \times 3=9]$ fallacies is the relativistic principle of equivalence according to which the interaction and acceleration related to the motion of a pair of bodies can be replaced with those of the motion of another different pair simply because the two pairs have a common body to which the two different motions are attached monastically (as one motion).

Relativity theory uses the term "inertialmass" for $(\mathrm{m})$ in Newton's second law $[F=m a]$, and the term "gravitationalmass" for $(\mathrm{m})$ in the law of universal gravitation $\left[F=\left(G M / d^{2}\right) m\right]$ in which the freefall acceleration is $\left[a=G M / d^{2}\right]$. The theory speaks of a law of equality of inertial and gravitational masses of a single body [4]. Some questions will naturally arise in this connection: What is it that the alleged two masses have in common that justifies calling them "masses" and in what respect do they differ so as to account for their distinction? If mass appeared in (n) basic laws would it be significant to ascribe (n) distinct masses to a single body? Would an Etvöv be required to establish the equality of the (n) masses experimentally? The applicability of Newton's second law to "contact (inertial) forces" and of his universal law to "gravitational forces" constitutes a basis not for distinguishing two kinds of mass, but two kinds of force. The alleged law is an insignificant idea that is not founded on any basis nor does it serve any purpose, but is merely desired to serve with the equivalence principle as a basis for general relativity.

2) Except the freefall acceleration, all the mechanical phenomena in the terrestrial vicinity are associated with the presence or absence of contact forces irrespective of the presence or absence of gravity. They arise from contact forces even in cases where these forces themselves arise from gravity. However it is a common incorrect practice to overlook the relevant contact force and have irrelevant gravity stressed in mind, such that these effects are attributed not to the presence and absence of the contact forces where they actually belong, but to the presence 
and absence of irrelevant gravity, thereby creating the "fallacies of misrelated causalities". Even in the branches of physics of most recent origin such as aviation and space flight one comes across such fallacies as "escape from gravity" and "disappearance of gravity". Examples of misrelated causalities are weight, weighing, pendulum oscillation, the concept of inertia, buldge of rotating bodies, ...

3) In certain conditions "quantummechanics" reduces to "classical mechanics" as a special limiting case. But there are "misplaced properties" so radically contradictory to classical mechanics that the two cannot be both correct. The interested thinker can, of course, revise classical mechanics to the extent related to the "misplaced properties" such as "existence of the non-existing", and "non-existence of the existing (gravity and acceleration in the equivalence principle)", "space without matter, and matter without space", "space traversing space, and time elapsing time", "action of matter on, or by non-matter"... such contradictions do require revision, and elimination of the associated fallacies.

4) In the special and general relativity theories one comes across some ideas such as (the equivalence principle, a body with two masses, space in a state of motion and furthermore contracting with motion, a time in a state of motion and moreover dilating with motion, the twin paradox, space and time existing in a continuum inseparably from one another but separately from matter, spacetime continuum undergoing curvature by matter and exerting geodesic (inertial) motion of matter by its curvature with the concept of force abolished, ...) which ideas appear as if they were science fiction. We touched only slightly on some of these concepts, but we have established beyond any doubt the untrueness of the equivalence principle. Since the refuted principle lies at the core of general relativity then "excluding the influence of prestige" and "casting Mach's doubts from time to time" on these concepts suggest themselves strongly. We hope that the concerned researchers will be attentive to the necessity of subjecting these concepts to revision, and to directing their research activities in these directions.

As already implied in the Extract, the conclusions mentioned above are introduced not as final truths but rather as opinions worthy of revision, and consequently of confirmation or refutation by other thinkers. These conclusions are extended in this work as an invitation for scientific debates on the topics dealt with in this work. However, this should in no way imply that we are not certain about the trueness of the conclusions reached. Posing these conclusions as unfinished opinions to be revised by readers generally is intended to reveal, on the one hand, a desire to allow a sufficient extent for revising such deeply lodged fallacies that are not easy to uproot readily. On the other hand, they are intended to avoid the possibility of being misinterpreted as violating the prestige of some highly rebutted scientists when they are actually intended as a continuation of their efforts and, as already mentioned, as an integration with these efforts by 
way of corrections, amendments and additions.

\section{References}

[1] Rindler, W. (1969) Essential Relativity. Van Nostrand Reinhold Company, New York. https://doi.org/10.1007/978-1-4757-1135-6

[2] Grigoryev, V. and Myakishev, G. (1971) The Forces of Nature. Mir Publishers. Moscow.

[3] Eddington, A.S. (1965) The Mathematical Theory of Relativity. Chelssea Publishing Company, New York.

[4] Einstein, A. and Infeld, L. (1971) The Evolution of Physics. Cambridge University Press, Cambridge.

[5] Einstein, A. (1977) Relativity. Methuen and Company Ltd., London.

[6] Allen Hynek, J. (1962) Challenge of the Universe. Scholastic Book Services, New York.

[7] Bernstein, J. (1978) Einstein. Fontana Collins, Glasgow.

Submit or recommend next manuscript to OALib Journal and we will provide best service for you:

- Publication frequency: Monthly

- 9 subject areas of science, technology and medicine

- Fair and rigorous peer-review system

- Fast publication process

- Article promotion in various social networking sites (LinkedIn, Facebook, Twitter, etc.)

- Maximum dissemination of your research work

Submit Your Paper Online: Click Here to Submit

Or Contact service@oalib.com 